\title{
ON THE DEPTH OF THE ASSOCIATED GRADED RING
}

\author{
ANNA GUERRIERI
}

(Communicated by Wolmer V. Vasconcelos)

\begin{abstract}
Let $(R, m)$ be a Cohen-Macaulay local ring of positive dimension $d$, let $I$ be an $m$-primary ideal of $R$. In this paper we individuate some conditions on $I$ that allow us to determine a lower bound for depth $\operatorname{gr}_{I}(R)$. It is proved that if $J \subseteq I$ is a minimal reduction of $I$ such that $\lambda\left(I^{2} \cap J / I J\right)=$ 2 and $I^{n} \cap J=I^{n-1} J$ for all $n \geq 3$, then depth $\operatorname{gr}_{I}(R) \geq d-2$; let us remark that $\lambda$ denotes the length function.
\end{abstract}

\section{INTRODUCTION}

Let $(R, m)$ be a noetherian local ring of dimension $d$ and let $I$ be an ideal of $R$. The symbol $\operatorname{gr}_{I}(R)$ will denote the associated graded ring of $I$

$$
\operatorname{gr}_{I}(R)=R / I \oplus I / I^{2} \oplus \cdots \oplus I^{n} / I^{n+1} \oplus \cdots .
$$

It is well known that, in this setting, $\operatorname{gr}_{I}(R)$ is a noetherian ring of dimension $d$.

If $(R, m)$ is a regular local ring of dimension $d$, then $\mathrm{gr}_{m}(R)$ is a regular ring as well; a more difficult task is to obtain information about $\mathrm{gr}_{I}(R)$ when $R$ is not regular and $I$ is any ideal. In particular trying to estimate depth $\operatorname{gr}_{I}(R)$ is a problem that has often been considered during the past years. Let us remark that, here and in what follows, by depth $\operatorname{gr}_{I}(R)$ we mean depth $\left(\operatorname{gr}_{I}(R)\right)_{M}$, where $M$ is the unique maximal homogeneous ideal of $\mathrm{gr}_{I}(R)$. In 1978 Valabrega and Valla [VV] showed that if $(R, m)$ is a $d$-dimensional Cohen-Macaulay local ring with infinite residue field and $I$ an $m$-primary ideal of $R$, then depth $\operatorname{gr}_{I}(R)=d$ if and only if there exists $J$ a minimal reduction of $I$ such that $I^{k} \cap J=I^{k-1} J$ for all $k \geq 2$. We can reformulate this result by saying that, under the same hypotheses, depth $\operatorname{gr}_{I}(R)=d$ if and only if there exists $J$ a minimal reduction of $I$ such that $\sum_{k \geq 2} \lambda\left(\frac{I^{k} \cap J}{I^{k-1} J}\right)=0$, where $\lambda$ denotes the length function. Therefore one might hope that, even when it is different from zero, the value of $\sum_{k \geq 2} \lambda\left(\frac{I^{k} \cap J}{I^{k-1} J}\right)$ will be related to the value of depth $\operatorname{gr}_{I}(R)$. Working in this direction we showed in a previous paper that if $(R, m)$ is as above, $I$ is an $m$-primary ideal, and $J$ a minimal

Received by the editors December 14, 1992 and, in revised form, April 5, 1993.

1991 Mathematics Subject Classification. Primary 13A30.

The author was partially supported by a grant of Consiglio Nazionale delle Ricerche, Italy. 
reduction of $I$ such that $\sum_{k \geq 2} \lambda\left(\frac{I^{k} \cap J}{I^{k-1} J}\right)=1$, then depth $\operatorname{gr}_{I}(R)=d-1$ (see Theorem 3.2 in $[G])$. In the present paper we present a further result in the same line of thought. More precisely, let $(R, m)$ be a Cohen-Macaulay local ring of dimension $d>0$ and with infinite residue field. Let $I$ be an $m$-primary ideal of $R$ and $J$ a minimal reduction of $I$ such that $\lambda\left(\frac{I^{2} \cap J}{I J}\right)=2$ and $I^{n} \cap J=I^{n-1} J$ for all $n \geq 3$. Then depth $\operatorname{gr}_{I}(R) \geq d-2$ (see Theorem 2.2).

In light of the above results, we conjecture that if $(R, m)$ is a CohenMacaulay local ring of dimension $d>0$ and with infinite residue field, $I$ is an $m$ - primary ideal, and $J$ a minimal reduction of $I$ such that $\sum_{k \geq 2} \lambda\left(\frac{I^{k} \cap J}{I^{k-1} J}\right)=$ $t$, then depth $\operatorname{gr}_{I}(R) \geq d-t$.

It appears clear from several recent works that being able to estimate a lower bound for depth $\operatorname{gr}_{I}(R)$ can be extremely fruitful. We refer to the results of Huckaba [Hu], Trung [T], Marley [M], and Sally [S2, S3], for the case depth $\operatorname{gr}_{I}(R) \geq d-1$. When depth $\operatorname{gr}_{I}(R) \geq d-2$ we would like to mention the recent results attained by Yinghwa Wu [W]. In her work depth $\operatorname{gr}_{I}(R) \geq d-2$ is the key assumption that allows her to acquire information about the reduction number of an $m$-primary ideal in a Cohen-Macaulay local ring.

In the next section we briefly describe the background notions that are necessary in our work, we proceed to proving our main result, and we use it to give a different proof of a recent result due to Vasconcelos [Vas].

\section{MAin RESUlt}

The techniques we employ are based on the notion, initially introduced by Northcott and Rees [NR], of reduction of an ideal. Let us recall here some definitions and facts concerning reductions. Given $(R, m)$ a noetherian local ring and $I$ a proper ideal of $R$, an ideal $J \subseteq I$ is a reduction of $I$ if $J I^{n}=I^{n+1}$ for some nonnegative integer $n$; we say that $J$ is a minimal reduction of $I$ if it is minimal among the reductions of $I$. It follows immediately that if $J$ is a reduction of $I$, then $I$ and $J$ have the same radical; in particular if $I$ is $m$-primary and $J$ is a reduction of $I, J$ is $m$-primary as well. In [NR] it was proven that every ideal $I$ contains a minimal reduction; furthermore, assuming $|R / m|=\infty$, any minimal base for a minimal reduction of $I$ has cardinality equal to $\operatorname{dim} \operatorname{gr}_{I}(R) \otimes R / m$. Therefore, if $I$ is an $m$-primary ideal of a noetherian local ring with infinite residue field, any minimal reduction of $I$ is minimally generated by a system of parameters. If in addition we have a Cohen-Macaulay local ring, we obtain that the elements of a minimal base of any minimal reduction of $I$ form a regular sequence. In what follows we will use repeatedly the above fact and all the proofs will be based on manipulations of regular sequences.

Besides we recall here a technical result that we proved in $[G]$, and that it will become useful later on (see Lemma 2.1 in [G]).

Lemma. Let $(R, m)$ be a Cohen-Macaulay local ring of dimension $d>0$ with infinite residue field, $I$ an m-primary ideal, and $J$ a minimal reduction of $I$. Let $\left\{x_{1}, \ldots, x_{d}\right\}$ be a minimal base for $J$ such that for some index $i, 1 \leq$ $i \leq d, I^{n} \cap\left(x_{1}, \ldots, \check{x}_{i}, \ldots, x_{d}\right) \subseteq I^{n-1} J$ for every $n, 1 \leq n \leq k$, with $k a$ positive integer. Then $I^{n} \cap\left(x_{1}, \ldots, \check{x}_{i}, \ldots, x_{d}\right)=I^{n-1}\left(x_{1}, \ldots, \check{x}_{i}, \ldots, x_{d}\right)$ for every $n, 1 \leq n \leq k$. 
We proceed now to the proof of a lemma which actually represents the central part of our principal result.

Lemma 2.1. Let $(R, m)$ be a Cohen-Macaulay local ring of dimension $d \geq 3$ with infinite residue field. Let $I$ be an m-primary ideal and $J$ a minimal reduction of I such that

$$
\lambda\left(\frac{I^{2} \cap J}{I J}\right)=2 .
$$

Then there exists $\left\{x_{1}, \ldots, x_{d}\right\}$, a minimal base for $J$, such that

$$
\lambda\left(\frac{I^{2} \cap J}{I J+I^{2} \cap\left(x_{1}, \ldots, \check{x}_{i}, \ldots, x_{d}\right)}\right) \neq 1
$$

for some integer $i, 1 \leq i \leq d$.

Before starting with the actual proof of Lemma 2.1, we prove the following statement.

Claim. Under the same hypotheses of Lemma 2.1, assume that given any minimal base $\left\{x_{1}, \ldots, x_{d}\right\}$ for $J$,

$$
\lambda\left(\frac{I^{2} \cap J}{I J+I^{2} \cap\left(x_{1}, \ldots, \check{x}_{i}, \ldots, x_{d}\right)}\right)=1
$$

for all integers $i, 1 \leq i \leq d$. Let $x$ be an element of $J-m J$ such that $I^{2} \cap(x) \neq I(x)$. Then, for each $y \in J-(m J+(x)), I^{2} \cap(y)=I(y)$.

Proof of Claim. Let $x$ be an element of $J-m J$ such that $I^{2} \cap(x) \neq I(x)$. This condition is equivalent to saying that $I^{2} \cap(x) \subseteq I J$. In fact, let $x_{2}, \ldots, x_{d}$ be elements of $J$ such that $\left\{x, x_{2}, \ldots, x_{d}\right\}$ forms a minimal base for $J$. If $I^{2} \cap$ $(x) \subseteq I J=I\left(x, x_{2}, \ldots, x_{d}\right)$, then $I^{2} \cap(x)=I(x)+I\left(x_{2}, \ldots, x_{d}\right) \cap(x)$. Since $\left\{x, x_{2}, \ldots, x_{d}\right\}$ is a regular sequence in $R$, we have that $I\left(x_{2}, \ldots, x_{d}\right) \cap(x)=$ $\left(x_{2}, \ldots, x_{d}\right)(x)$ and consequently we would obtain $I^{2} \cap(x)=I(x)$. Therefore the assumption $I^{2} \cap(x) \neq I(x)$ implies that $\lambda\left(\frac{I^{2} \cap J}{I J+I^{2} \cap(x)}\right)<\lambda\left(\frac{I^{2} \cap J}{I J}\right)=2$. Indeed we are going to prove that $\lambda\left(\frac{I^{2} \cap J}{I J+I^{2} \cap(x)}\right)=1$. If $\lambda\left(\frac{I^{2} \cap J}{I J+I^{2} \cap(x)}\right)=0$, then $I^{2} \cap J=I J+I^{2} \cap(x)$ and, given $x_{2}, \ldots, x_{d}$ in $J$ such that $\left\{x, x_{2}, \ldots, x_{d}\right\}$ is a minimal base for $J$, we have that $I^{2} \cap\left(x_{2}, \ldots, x_{d}\right) \subseteq I^{2} \cap J=I J+I^{2} \cap(x)$. Let $\alpha_{2} x_{2}+\cdots+\alpha_{d} x_{d}$ be an element of $I^{2} \cap\left(x_{2}, \ldots, x_{d}\right)$, then one can write $\alpha_{2} x_{2}+\cdots+\alpha_{d} x_{d}=i_{2} x_{2}+\cdots+i_{d} x_{d}+r x$ where $i_{j} \in I$ for all $j=2, \ldots, d$ and $r x \in I^{2} \cap(x)$. Since $\left\{x, x_{2}, \ldots, x_{d}\right\}$ is a regular sequence in $R$, we deduce that the coefficients $\alpha_{j}$ are in $I$ for all $j=2, \ldots, d$ and we conclude that $I^{2} \cap\left(x_{2}, \ldots, x_{d}\right)=I\left(x_{2}, \ldots, x_{d}\right)$. This contradicts the assumption

$$
\lambda\left(\frac{I^{2} \cap J}{I J+I^{2} \cap\left(x_{2}, \ldots, x_{d}\right)}\right)=1 .
$$

Therefore we obtain

$$
\lambda\left(\frac{I^{2} \cap J}{I J+I^{2} \cap(x)}\right)=1 .
$$

Let $y \in J-(m J+(x))$ and let $x_{3}, \ldots, x_{d}$ be elements in $J$ such that $\left\{x, y, x_{3}, \ldots, x_{d}\right\}$ constitutes a minimal base for $J$. By our assumptions 
we have

$$
\lambda\left(\frac{I^{2} \cap J}{I J+I^{2} \cap(x)}\right)=\lambda\left(\frac{I^{2} \cap J}{I J+I^{2} \cap\left(x, y, x_{3}, \ldots, \check{x}_{i}, \ldots, x_{d}\right)}\right)=1
$$

for all $i=3, \ldots, d$. Thus we have $I^{2} \cap(y) \subseteq I^{2} \cap\left(x, y, x_{3}, \ldots, \check{x}_{i}, \ldots, x_{d}\right)$ $\subseteq I J+I^{2} \cap(x)$ and we can derive from it that $I^{2} \cap(y)=I(y)+\left(I\left(x_{3}, \ldots, x_{d}\right)+\right.$ $\left.I^{2} \cap(x)\right) \cap(y)$. Let $\alpha y$ be an element of $\left(I\left(x_{3}, \ldots, x_{d}\right)+I^{2} \cap(x)\right) \cap(y)$, we can write $\alpha y=i_{3} x_{3}+\cdots+i_{d} x_{d}+r x$ with $i_{j} \in I$ for all $j=3, \ldots, d$ and $r x \in$ $I^{2} \cap(x)$. Since $\left\{x, y, x_{3}, \ldots, x_{d}\right\}$ is a regular sequence, $\alpha \in\left(x, x_{3}, \ldots, x_{d}\right)$ and $\left(I\left(x_{3}, \ldots, x_{d}\right)+I^{2} \cap(x)\right) \cap(y)=\left(x, x_{3}, \ldots, x_{d}\right)(y)$. In conclusion we obtain $I^{2} \cap(y)=I(y)$.

Proof of Lemma 2.1. We will proceed by induction on $d$ and by contradiction. We begin with induction on $d$.

Case $d=3$. Suppose, by contradiction, that given any minimal base $\left\{x_{1}\right.$, $\left.x_{2}, x_{3}\right\}$ for $J$,

$$
\lambda\left(\frac{I^{2} \cap J}{I J+I^{2} \cap\left(x_{i}, x_{j}\right)}\right)=1 \text { for all } i \text { and } j, 1 \leq i \neq j \leq 3 .
$$

The claim previously proved asserts that either $I^{2} \cap(x)=I(x)$ for each $x$ in $J-m J$, or if there exists an $x \in J-m J$ with $I^{2} \cap(x) \neq I(x)$, then $I^{2} \cap(y)=I(y)$ for each $y \in J-(m J+(x))$. Even if such an element $x$ exists we can always choose $\left\{x_{1}, x_{2}, x_{3}\right\}$ a minimal base for $J$ such that also $\left\{x, x_{i}, x_{j}\right\}$ is a minimal base for $J$ for all $i$ and $j, 1 \leq i \neq j \leq 3$. In particular, $I^{2} \cap\left(x_{i}\right)=I\left(x_{i}\right)$ for all $i, 1 \leq i \leq 3$. By assumption

$$
\begin{aligned}
\lambda\left(\frac{I^{2} \cap J}{I^{2} \cap\left(x_{1}, x_{2}\right)+I J}\right) & =\lambda\left(\frac{I^{2} \cap J}{I^{2} \cap\left(x_{1}, x_{3}\right)+I J}\right) \\
& =\lambda\left(\frac{I^{2} \cap J}{I^{2} \cap\left(x_{2}, x_{3}\right)+I J}\right)=1 .
\end{aligned}
$$

Therefore, we have

$$
\begin{aligned}
\lambda\left(\frac{I^{2} \cap\left(x_{1}, x_{2}\right)+I J}{I J}\right) & =\lambda\left(\frac{I^{2} \cap\left(x_{1}, x_{3}\right)+I J}{I J}\right) \\
& =\lambda\left(\frac{I^{2} \cap\left(x_{2}, x_{3}\right)+I J}{I J}\right)=1,
\end{aligned}
$$

and we can write

$$
\begin{aligned}
I^{2} \cap\left(x_{1}, x_{2}\right)+I J & =I J+\left(a_{1} x_{1}+a_{2} x_{2}\right), \\
& a_{1} x_{1}+a_{2} x_{2} \notin I J, m\left(a_{1} x_{1}+a_{2} x_{2}\right) \subseteq I J ; \\
I^{2} \cap\left(x_{1}, x_{3}\right)+I J= & I J+\left(b_{1} x_{1}+b_{3} x_{3}\right), \\
& b_{1} x_{1}+b_{3} x_{3} \notin I J, m\left(b_{1} x_{1}+b_{3} x_{3}\right) \subseteq I J ; \\
I^{2} \cap\left(x_{2}, x_{3}\right)+I J= & I J+\left(c_{2} x_{2}+c_{3} x_{3}\right), \\
& c_{2} x_{2}+c_{3} x_{3} \notin I J, m\left(c_{2} x_{2}+c_{3} x_{3}\right) \subseteq I J .
\end{aligned}
$$

It is not difficult to derive that the coefficient $a_{1}$ does not belong to the ideal $I$ since $I^{2} \cap\left(x_{2}\right)=I\left(x_{2}\right)$ while $a_{1} x_{1}+a_{2} x_{2} \notin I J$. Similarly one obtains that $a_{2}, b_{1}, b_{2}, c_{2}, c_{3} \notin I$. Thus $I^{2} \cap\left(x_{2}, x_{3}\right)+I J$ is neither con- 
tained in $I^{2} \cap\left(x_{1}, x_{2}\right)+I J$ nor in $I^{2} \cap\left(x_{1}, x_{3}\right)+I J$. For example, suppose $I^{2} \cap\left(x_{2}, x_{3}\right)+I J \subseteq I^{2} \cap\left(x_{1}, x_{2}\right)+I J$. Then $c_{2} x_{2}+c_{3} x_{3}$ would be an element of $I^{2} \cap\left(x_{1}, x_{2}\right)+I J$ and we would be able to write $c_{2} x_{2}+c_{3} x_{3}=i_{3} x_{3}+r_{1} x_{1}+r_{2} x_{2}$ with $i_{3} \in I$ and $r_{1} x_{1}+r_{2} x_{2} \in I^{2}$. Since $\left\{x_{1}, x_{2}, x_{3}\right\}$ is a regular sequence, we would obtain $c_{3} \in I$, the situation that we just ruled out. By assumption,

$$
\lambda\left(\frac{I^{2} \cap J}{I^{2} \cap\left(x_{1}, x_{2}\right)+I J}\right)=1,
$$

thus we can write $I^{2} \cap J=I^{2} \cap\left(x_{1}, x_{2}\right)+I J+(\omega)$ with $\omega \notin I^{2} \cap\left(x_{1}, x_{2}\right)+I J$ and $m \omega \subseteq I^{2} \cap\left(x_{1}, x_{2}\right)+I J$. Because of what we have just said, we can take $\omega=c_{2} x_{2}+c_{3} x_{3}$. In this way it is possible to write the expression $I^{2} \cap J=$ $I J+I^{2} \cap\left(x_{1}, x_{2}\right)+I^{2} \cap\left(x_{2}, x_{3}\right)=I J+\left(a_{1} x_{1}+a_{2} x_{2}, c_{2} x_{2}+c_{3} x_{3}\right)$. Consider now the set $\left\{x_{3}, x_{1}+x_{2}, x_{3}+x_{2}\right\}$. It is a minimal base for $J$, and $x_{1}+x_{2}$ and $x_{3}+x_{2}$ are both in $J-(m J+(x))$ since $\left\{x, x_{1}, x_{2}\right\}$ and $\left\{x, x_{2}, x_{3}\right\}$ are both minimal bases for $J$. By repeating all the considerations made above, we conclude that

$$
I^{2} \cap J=I J+\left(\alpha\left(x_{1}+x_{2}\right)+\beta x_{3}, \alpha^{\prime}\left(x_{1}+x_{2}\right)+\beta^{\prime}\left(x_{3}+x_{2}\right)\right)
$$

with $\alpha\left(x_{1}+x_{2}\right)+\beta x_{3}, \alpha^{\prime}\left(x_{1}+x_{2}\right)+\beta^{\prime}\left(x_{3}+x_{2}\right) \notin I J$ while $m\left(\alpha\left(x_{1}+x_{2}\right)+\beta x_{3}\right)$ and $m\left(\alpha^{\prime}\left(x_{1}+x_{2}\right)+\beta^{\prime}\left(x_{3}+x_{2}\right)\right)$ are both contained in $I J$. As before we have $\alpha, \beta, \alpha^{\prime}, \beta^{\prime} \notin I$. Since $I^{2} \cap J=I J+\left(a_{1} x_{1}+a_{2} x_{2}, c_{2} x_{2}+c_{3} x_{3}\right)$ too, we can write

$$
\begin{aligned}
& \alpha\left(x_{1}+x_{2}\right)+\beta x_{3} \equiv \theta_{1}\left(a_{1} x_{1}+a_{2} x_{2}\right)+\theta_{2}\left(c_{2} x_{2}+c_{3} x_{3}\right), \\
& \alpha^{\prime}\left(x_{1}+x_{2}\right)+\beta^{\prime}\left(x_{3}+x_{2}\right) \equiv \theta_{1}^{\prime}\left(a_{1} x_{1}+a_{2} x_{2}\right)+\theta_{2}^{\prime}\left(c_{2} x_{2}+c_{3} x_{3}\right),
\end{aligned}
$$

where we are taking congruences modulo $I J$. Looking at the first congruence and knowing that $\left\{x_{1}, x_{2}, x_{3}\right\}$ is a regular sequence in $R$, we immediately get the relations $\alpha-\theta_{1} a_{1} \in I, \quad \alpha-\theta_{1} a_{2}-\theta_{2} c_{2} \in I$, and $\beta-\theta_{2} c_{3} \in I$ and we obtain $\theta_{1}\left(a_{1}-a_{2}\right)-\theta_{2} c_{2} \in I$. Repeating the same procedure for the second congruence we find relations analogous to those we found before, namely $\alpha^{\prime}-\theta_{1}^{\prime} a_{1} \in I$, $\alpha^{\prime}+\beta^{\prime}-\theta_{1}^{\prime} a_{2}-\theta_{2}^{\prime} c_{2} \in I$, and $\beta^{\prime}-\theta_{2}^{\prime} c_{3} \in I$. In this way $\theta_{1}^{\prime}\left(a_{1}-a_{2}\right)+\theta_{2}^{\prime}\left(c_{3}-c_{2}\right) \in$ I. Gathering all the information, we obtain that $\theta_{1}\left(a_{1}-a_{2}\right)-\theta_{2} c_{2} \in I$ and $\theta_{1}^{\prime}\left(a_{1}-a_{2}\right)-\theta_{2}^{\prime}\left(c_{3}-c_{2}\right) \in I$. A simple computation allow us to conclude that the element $\left(\theta_{2} \theta_{1}^{\prime}-\theta_{1} \theta_{2}^{\prime}\right) c_{2}+\theta_{1} \theta_{2}^{\prime} c_{3}$ is in the ideal $I$. From this we can now deduce that the element $\theta_{2} \theta_{1}^{\prime}-\theta_{1} \theta_{2}^{\prime}$ belongs to the maximal ideal $m$. If $\theta_{2} \theta_{1}^{\prime}-\theta_{1} \theta_{2}^{\prime}$ were a unit $u$, we would have $u c_{2}+v c_{3} \in I$, with $v=\theta_{1} \theta_{2}^{\prime}$. Therefore, $c_{2}=i-u^{-1} v c_{3}$ with $i \in I$ and $c_{2} x_{2}+c_{3} x_{3}=\left(i-u^{-1} v c_{3}\right) x_{2}+$ $c_{3} x_{3}=i x_{2}+c_{3}\left(x_{3}-u^{-1} v x_{2}\right)$. Since $c_{2} x_{2}+c_{3} x_{3}$ and $i x_{2}$ are both in $I^{2}$ and $x_{3}-u^{-1} v x_{2}$ belongs to $J-(m J+(x))$ because $\left\{x, x_{2}, x_{3}\right\}$ is a minimal base for $J$, we obtain $c_{3}\left(x_{3}-u^{-1} v x_{2}\right) \in I^{2} \cap\left(x_{3}-u^{-1} v x_{2}\right)=I\left(x_{3}-u^{-1} v x_{2}\right)$. In this way the coefficient $c_{3}$ would be in $I$ which contradicts our assumptions. Hence $\theta_{2} \theta_{1}^{\prime}-\theta_{1} \theta_{2}^{\prime}$ belongs to $m$ and this is a contradiction since the elements $\alpha\left(x_{1}+x_{2}\right)+\beta x_{3}$ and $\alpha^{\prime}\left(x_{1}+x_{2}\right)+\beta^{\prime}\left(x_{3}+x_{2}\right)$ are linearly independent over the residue field $R / m$ once we go $\bmod I J$. In this way we proved that the statement is correct in dimension $d=3$.

Case $d>3$. Suppose the statement true for all rings satisfying our hypotheses and having dimension $d^{\prime}, 3 \leq d^{\prime}<d$. We want to show that the statement is true for any ring, satisfying our hypotheses, with dimension $d$. Let us proceed, 
once again, by contradiction. We are supposing that

$$
\lambda\left(\frac{I^{2} \cap J}{I J+I^{2} \cap\left(x_{1}, \ldots, \check{x}_{i}, \ldots, x_{d}\right)}\right)=1
$$

for any minimal base, $\left\{x_{1}, \ldots, x_{d}\right\}$, for $J$ and for any integer $i$, with $1 \leq$ $i \leq d$. Once again by the Claim proved at the beginning, either $I^{2} \cap(x)=I(x)$ for each $x$ in $J-m J$ or, if there exists an $x \in J-m J$ with $I^{2} \cap(x) \neq I(x)$, then $I^{2} \cap(y)=I(y)$ for each $y \in J-(m J+(x))$. Let us fix, now, a minimal base for $J, J=\left(x_{1}, \ldots, x_{d}\right)$. Without loss of generality we may assume that $I^{2} \cap\left(x_{i}\right)=I\left(x_{i}\right)$ for all $i, 1 \leq i \leq d$. Since in particular we have $I^{2} \cap\left(x_{d}\right)=I\left(x_{d}\right)$, the quotient ring $\bar{R}=R /\left(x_{d}\right)$ is a Cohen-Macaulay local ring with dimension $d-1, \bar{I}=I /\left(x_{d}\right)$ is an $\bar{m}$-primary ideal of $\bar{R}$, and $\bar{J}=J /\left(x_{d}\right)$ is a minimal reduction of $\bar{I}$ with $\lambda\left(\frac{\bar{I}^{2} \cap \bar{J}}{\overline{I J}}\right)=2$. By inductive hypothesis there exists a minimal set of generators for $\bar{J}, \bar{J}=\left(\bar{f}_{1}, \ldots, \bar{f}_{d-1}\right)$, such that

$$
\lambda\left(\frac{\bar{I}^{2} \cap \bar{J}}{\overline{I J}+\bar{I}^{2} \cap\left(\overline{f_{1}}, \ldots, \overline{\bar{f}}_{i}, \ldots, \bar{f}_{d-1}\right)}\right) \neq 1 \text { for some } i, \quad 1 \leq i \leq d-1 \text {. }
$$

Therefore $\left\{f_{1}, \ldots, f_{d-1}, x_{d}\right\}$ is a minimal set of generators for $J$ such that

$$
\begin{gathered}
\frac{I^{2} \cap J}{I J+I^{2} \cap\left(f_{1}, \ldots, \check{f}_{i}, \ldots, f_{d-1}, x_{d}\right)} \\
\cong \frac{\bar{I}^{2} \cap \bar{J}}{\overline{I J}+\bar{I}^{2} \cap\left(\overline{f_{1}}, \ldots, \check{\bar{f}}_{i}, \ldots, \bar{f}_{d-1}\right)} .
\end{gathered}
$$

Since the length of the latter is different from 1 , we have a contradiction. Hence the statement must be true in any dimension $d$.

Now we are able to prove the main result.

Theorem 2.2. Let $(R, m)$ be a Cohen-Macaulay local ring of dimension $d>0$ with infinite residue field, $I$ an $m$-primary ideal, and $J$ a minimal reduction of $I$ such that

$$
\lambda\left(\frac{I^{2} \cap J}{I J}\right)=2 \text { and } I^{n} \cap J=I^{n-1} J \text { for each } n \geq 3 .
$$

Then depth $\operatorname{gr}_{I}(R) \geq d-2$.

Proof. Since the statement is clearly satisfied when $d \leq 2$, we proceed by induction on $d$. Assume $d \geq 3$ and the statement true for rings of dimension strictly less than $d$. By the lemma previously proved there exists $\left\{x_{1}, \ldots, x_{d}\right\}$, a minimal base for $J$, such that for some $i, 1 \leq i \leq d$,

$$
\lambda\left(\frac{I^{2} \cap J}{I J+I^{2} \cap\left(x_{2}, \ldots, \check{x}_{i}, \ldots, x_{d}\right)}\right) \neq 1 .
$$

Without loss of generality we can assume that $i=d$ and we obtain that

$$
\lambda\left(\frac{I^{2} \cap J}{I J+I^{2} \cap\left(x_{1}, \ldots, x_{d-1}\right)}\right)
$$


has either value 0 or value 2 . We will analyze the two cases separatedly.

Case 1. Let us assume

$$
\lambda\left(\frac{I^{2} \cap J}{I J+I^{2} \cap\left(x_{1}, \ldots, x_{d-1}\right)}\right)=0 .
$$

This is equivalent to saying that $I^{2} \cap J=I J+I^{2} \cap\left(x_{1}, \ldots, x_{d-1}\right)=I\left(x_{d}\right)+I^{2} \cap$ $\left(x_{1}, \ldots, x_{d-1}\right)$. Consequently we have $I^{2} \cap\left(x_{d}\right) \subseteq I\left(x_{d}\right)+I^{2} \cap\left(x_{1}, \ldots, x_{d-1}\right)$ from which one can easily deduce that $I^{2} \cap\left(x_{d}\right)=I\left(x_{d}\right)+I^{2} \cap\left(x_{1}, \ldots, x_{d-1}\right) \cap$ $\left(x_{d}\right)$. Since $\left\{x_{1}, \ldots, x_{d}\right\}$ is a regular sequence in $R,\left(x_{1}, \ldots, x_{d-1}\right) \cap\left(x_{d}\right)=$ $\left(x_{1}, \ldots, x_{d-1}\right)\left(x_{d}\right)$ and we obtain $I^{2} \cap\left(x_{d}\right)=I\left(x_{d}\right)$. Since $x_{d} \in I-I^{2}$, its leading form in $\operatorname{gr}_{I}(R)$ is the image of $x_{d}$ itself in $I / I^{2}$. We claim that, in the present situation, the leading form of $x_{d}$ is a regular element in $\operatorname{gr}_{I}(R)$. By the results of Valabrega and Valla [VV] we need to show that $I^{n} \cap\left(x_{d}\right)=I^{n-1}\left(x_{d}\right)$ for every $n \geq 2$. We already know that $I^{2} \cap\left(x_{d}\right)=I\left(x_{d}\right)$, and we have that $I^{n} \cap\left(x_{d}\right) \subseteq I^{n-1} J$ for every $n \geq 2$. This will allow us to show what we want once we prove the next Claim .

Claim. Let $(R, m)$ be a noetherian local ring. Let $I$ and $J$ be ideals of $R$ such that $J$ is minimally generated by regular sequence $\left\{x_{1}, \ldots, x_{d}\right\}, J \subseteq I$, and we have the equalities $I^{2} \cap J=I J+I^{2} \cap\left(x_{1}, \ldots, x_{d-1}\right)$ and $I^{n} \cap J=I^{n-1} J$ for all $n \geq 3$. Then, given any positive integer $n, I^{t}\left(x_{1}, \ldots, x_{d-1}\right)^{n-t} \cap\left(x_{d}\right) \subseteq$ $I^{n-1}\left(x_{d}\right)$ for all $t$ with $0 \leq t \leq n-1$.

Remark. In the proof of this statement we will use a result due to Kaplansky [K]. Namely, let $(R, m)$ be a local ring and $x_{1}, \ldots, x_{s}$ be elements constituting a regular sequence in $R$. Let $H$ be an ideal generated by monomials in $x_{2}, \ldots, x_{s}$. Then $t x_{1} \in H$ implies $t \in H$.

Proof of Claim. We will proceed by induction on $t$. Let $t \leq 1$. Let $r x_{d}$ be an element of $I^{t}\left(x_{1}, \ldots, x_{d-1}\right)^{n-t}$, in particular $r x_{d} \in\left(x_{1}, \ldots, x_{d-1}\right)^{n-t}$ and, using the above remark, we obtain $r \in\left(x_{1}, \ldots, x_{d-1}\right)^{n-t}$. Consequently $r x_{d} \in\left(x_{1}, \ldots, x_{d-1}\right)^{n-t}\left(x_{d}\right)$ which is a subset of $I^{n-t}\left(x_{d}\right)$ with $t$ an integer either equal to 0 or equal to 1 . In either case we obtain what we want. Assume $t \geq 2$, and let $r x_{d}$ be an element of $I^{t}\left(x_{1}, \ldots, x_{d-1}\right)^{n-t}$; once again we can conclude that $r \in\left(x_{1}, \ldots, x_{d-1}\right)^{n-t}$. Therefore we can write the expression,

$$
r=\sum_{\substack{|\Lambda|=n-t \\ \Lambda=\left\{\lambda_{1}, \ldots, \lambda_{d-1}\right\}}} r_{\Lambda} x_{1}^{\lambda_{1}} \cdots x_{d-1}^{\lambda_{d-1}} .
$$

Since the element $r x_{d}$ belongs to $I^{t}\left(x_{1}, \ldots, x_{d-1}\right)^{n-t}$ too, we obtain the following equalities

$$
\begin{aligned}
& \sum_{\substack{\Lambda,|\Lambda|=n-t \\
\Lambda=\left\{\lambda_{1}, \ldots, \lambda_{d-1}\right\}}} r_{\Lambda} x_{d} x_{1}^{\lambda_{1}} \cdots x_{d-1}^{\lambda_{d-1}}=r x_{d} \\
& \quad=\sum_{\substack{|\Lambda|=n-t \\
\Lambda=\left\{\lambda_{1}, \ldots, \lambda_{d-1}\right\}}} i_{\Lambda} x_{1}^{\lambda_{1}} \cdots x_{d-1}^{\lambda_{d-1}}, \quad i_{\Lambda} \in I^{t} \forall \Lambda .
\end{aligned}
$$

As $\left\{x_{1}, \ldots, x_{d}\right\}$ is a regular sequence in $R$, by equating coefficients in the previous expressions, we get $r_{\Lambda} x_{d}-i_{\Lambda} \in\left(x_{1}, \ldots, x_{d-1}\right)$ for every index $\Lambda$. 
Let us distinguish two cases. If $t=2$ we have $i_{\Lambda} \in I^{2} \cap\left(x_{1}, \ldots, x_{d}\right)=$ $I J+I^{2} \cap\left(x_{1}, \ldots, x_{d-1}\right)=I\left(x_{d}\right)+I^{2} \cap\left(x_{1}, \ldots, x_{d-1}\right)$ for every $\Lambda$. This implies that the element $r_{\Lambda} x_{d}$ belongs to $I\left(x_{d}\right)+I^{2} \cap\left(x_{1}, \ldots, x_{d-1}\right)+\left(x_{1}, \ldots, x_{d-1}\right)=$ $I\left(x_{d}\right)+\left(x_{1}, \ldots, x_{d-1}\right)$. Once again because $\left\{x_{1}, \ldots, x_{d}\right\}$ is a regular sequence in $R$, we get $r_{\Lambda} \in I$ for all $\Lambda$. Hence, going back to the first expression we wrote for $r$, we find that $r \in I I^{n-2}=I^{n-1}$ and $r x_{d} \in I^{n-1}\left(x_{d}\right)$ as wanted. If $t \geq 3$ we have $i_{\Lambda} \in I^{t} \cap\left(x_{1}, \ldots, x_{d}\right)=I^{t-1} J$ for every $\Lambda$. In this way we obtain

$$
\begin{aligned}
r x_{d} & \in I^{t-1} J\left(x_{1}, \ldots, x_{d-1}\right)^{n-t} \\
& =I^{t-1}\left(x_{1}, \ldots, x_{d-1}\right)^{n-t+1}+I^{t-1}\left(x_{d}\right)\left(x_{1}, \ldots, x_{d-1}\right)^{n-t} .
\end{aligned}
$$

Thus we have $I^{t}\left(x_{1}, \ldots, x_{d-1}\right)^{n-t} \cap\left(x_{d}\right) \subseteq I^{t-1}\left(x_{1}, \ldots, x_{d-1}\right)^{n-t+1} \cap\left(x_{d}\right)+$ $I^{n-1}\left(x_{d}\right)$. By applying the inductive hypothesis we get

$$
I^{t-1}\left(x_{1}, \ldots, x_{d-1}\right)^{n-t+1} \cap\left(x_{d}\right) \subseteq I^{n-1}\left(x_{d}\right) .
$$

This proves that $I^{t}\left(x_{1}, \ldots, x_{d-1}\right)^{n-t} \cap\left(x_{d}\right) \subseteq I^{n-1}\left(x_{d}\right)$ and concludes the proof of the claim.

We will use the previous claim to prove that $I^{n} \cap\left(x_{d}\right)=I^{n-1}\left(x_{d}\right)$ for each $n \geq 3$. Since $I^{n} \cap\left(x_{d}\right) \subseteq I^{n-1} J$, we get $I^{n} \cap\left(x_{d}\right)=I^{n-1}\left(x_{d}\right)+$ $I^{n-1}\left(x_{1}, \ldots, x_{d-1}\right) \cap\left(x_{d}\right)$. By using the Claim in the case $t=n-1$ we have $I^{n-1}\left(x_{1}, \ldots, x_{d-1}\right) \cap\left(x_{d}\right) \subseteq I^{n-1}\left(x_{d}\right)$. Hence we attain precisely $I^{n} \cap\left(x_{d}\right)=$ $I^{n-1}\left(x_{d}\right)$ for each $n \geq 3$. If we denote with the symbol $x_{d}^{*}$ the leading form of $x_{d}$ in $\operatorname{gr}_{I}(R)$, now we know that $x_{d}^{*}$ is a regular element. Therefore, passing to the quotient ring $R /\left(x_{d}\right)=\bar{R}$, we have the isomorphism $\operatorname{gr}_{\bar{I}}(\bar{R}) \cong \operatorname{gr}_{I}(R) / x_{d}^{*} \operatorname{gr}_{I}(R)$. Now $(\bar{R}, \bar{m})$ is a Cohen-Macaulay local ring of dimension $d-1>0$ and with infinite residue field. $\bar{I}$ is an $\bar{m}$-primary ideal of $\bar{R}$ and $\bar{J}$ is a minimal reduction of $\bar{I}$. Since $I^{n} \cap\left(x_{d}\right)=I^{n-1}\left(x_{d}\right)$ for all $n \geq 2$,

$$
\lambda\left(\frac{\bar{I}^{2} \cap \bar{J}}{\overline{I J}}\right)=\lambda\left(\frac{I^{2} \cap J}{I J}\right)=2, \quad \text { while } \quad \lambda\left(\frac{\bar{I}^{n} \cap \bar{J}}{\bar{I}^{n-1} \bar{J}}\right)=\lambda\left(\frac{I^{n} \cap J}{I^{n-1} J}\right)=0
$$

for all $n \geq 3$. Therefore all our hypotheses are satisfied by the ring $\bar{R}$ and, by induction, we conclude that depth $\operatorname{gr}_{\bar{I}}(\bar{R}) \geq(d-1)-2=d-3$. As $x_{d}^{*}$ is a regular element, depth $\operatorname{gr}_{I}(R) \geq d-2$.

Case 2. Let us assume

$$
\lambda\left(\frac{I^{2} \cap J}{I J+I^{2} \cap\left(x_{1}, \ldots, x_{d-1}\right)}\right)=2
$$

This implies that the module $\frac{I^{2} \cap J}{I J}$ is isomorphic to the module

$$
\frac{I^{2} \cap J}{I J+I^{2} \cap\left(x_{1}, \ldots, x_{d-1}\right)} \text {. }
$$

Thus we can deduce that $I^{2} \cap\left(x_{1}, \ldots, x_{d-1}\right) \subseteq I J$ and consequently $I^{n} \cap$ $\left(x_{1}, \ldots, x_{d-1}\right) \subseteq I^{n-1} J$ for every $n \geq 2$. Therefore, by Lemma 2.1 in [G], which we mentioned at the beginning,

$$
I^{n} \cap\left(x_{1}, \ldots, x_{d-1}\right)=I^{n-1}\left(x_{1}, \ldots, x_{d-1}\right)
$$


for every $n \geq 2$. Hence, we actually obtain that depth $\operatorname{gr}_{I}(R) \geq d-1$ in this case.

Remark. It is possible to remove the assumption $|R / m|=\infty$ by using the faithfully flat extension from $R$ to $R(X)=R[X]_{m R[X]}$. In fact, we can prove that if $(R, m)$ is a Cohen-Macaulay local ring of dimension $d>0, I$ an $m$-primary ideal of $R$, and $J$ a reduction of $I$ minimally generated by $d$ elements such that $\lambda\left(\frac{I^{2} \cap J}{I J}\right)=2$ and $I^{n} \cap J=I^{n-1} J$ for all $n \geq 3$, then depth $\operatorname{gr}_{I}(R) \geq d-2$.

It is now possible to use Theorem 2.2 and the result obtained in a previous work that was mentioned in the Introduction (see Theorem 3.2.in [G]) to give a different proof of the following result due to Vasconcelos [Vas].

Corollary 2.3. Let $(R, m)$ be a Cohen-Macaulay local ring of dimension $d>0$ and with infinite residue field. Let $I$ be an $m$-primary ideal and $J$ a minimal reduction of $I$ such that $I^{3}=I^{2} J$.

(a) If $I^{2} / I J \cong R / m$, then depth $\operatorname{gr}_{I}(R) \geq d-1$.

(b) If $I^{2} / I J \cong R / m \times R / m$, then depth $\mathrm{gr}_{I}(R) \geq d-2$.

Proof. Let us start by proving the case described in (a). Since $\frac{I^{n} \cap J}{I^{n-1} J}$ is a submodule of $\frac{I^{n}}{I^{n-1 J}}$ for all $n \geq 2$, the hypotheses imply that $\lambda\left(\frac{I^{2} \cap J}{I J}\right) \leq 1$ and $I^{n} \cap J=I^{n-1} J$ for all $n \geq 3$. Consequently, either $I^{n} \cap J=I^{n-1} J$ for all $n \geq 2$ or $\lambda\left(\frac{I^{2} \cap J}{I J}\right)=1$ and $I^{n} \cap J=I^{n-1} J$ for all $n \geq 3$. In the first case one has depth $\operatorname{gr}_{I}(R)=d$ by the results in [VV], in the second case we can use Theorem 3.2 in [G] and we obtain depth $\operatorname{gr}_{I}(R)=d-1$; in either case depth $\operatorname{gr}_{I}(R) \geq d-1$. In case (b) we proceed very much in the same way. Once again the hypotheses imply that $\lambda\left(\frac{I^{2} \cap J}{I J}\right) \leq 2$ and $I^{n} \cap J=I^{n-1} J$ for all $n \geq 3$. If $\lambda\left(\frac{I^{2} \cap J}{I J}\right) \leq 1$, we can repeat the proof given in (a). If $\lambda\left(\frac{I^{2} \cap J}{I J}\right)=2$, we can use Theorem 2.2 and we obtain depth $\operatorname{gr}_{I}(R) \geq d-2$; in all the cases depth $\operatorname{gr}_{I}(R) \geq d-2$.

\section{ACKNOWLEDGMENT}

I thank Craig Huneke for many valuable conversations concerning the material in this paper.

\section{REFERENCES}

[G] A. Guerrieri, On the depth of the associated graded ring of an $m$-primary ideal of a Cohen-Macaulay local ring, J. Algebra (to appear).

[Hu] S. Huckaba, Reduction numbers for ideals of higher analytic spread, Math. Proc. Cambridge Philos. Soc. 102 (1987), 49-57.

[H] C. Huneke, Hilbert functions and symbolic powers, Michigan Math. J. 34 (1987), 293-318.

[K] I. Kaplansky, R-sequences and homological dimension, Nagoya Math. J. (1962), 195-199.

[M] T. Marley, The coefficients of the Hilbert polynomial and the reduction number of an ideal, J. London Math. Soc. (2) 40 (1989), 1-8.

[Mat] H. Matsumura, Commutative ring theory, Cambridge Univ. Press, London and New York, 1990. 
[NR] D. G. Northcott and D. Rees, Reduction of ideals in local rings, Math. Proc. Cambridge Philos. Soc. 50 (1954), 145-158.

[S1] J. Sally, Reductions, local cohomology and Hilbert functions of local rings, Commutative Algebra (Durham 1981), London Math. Soc. Lecture Notes Ser., vol. 72, Cambridge Univ. Press, London, 1982, pp. 231-241.

[S2] _ Hilbert coefficients and reduction number 2, J. Algebraic Geom. 1 (1992), 325-333.

[S3] __ Ideals whose Hilbert function and Hilbert polynomial agree at $n=1$, preprint.

[S4] _ Numbers of generators of ideals in local rings, Lecture Notes in Pure and Appl. Math., vol. 35, Marcel Dekker, New York, 1978.

[T] N. V. Trung, Reduction exponent and degree bound for the defining equations of graded rings, Proc. Amer. Math. Soc. 101 (1987), 229-236.

[V] G. Valla, On form rings that are Cohen-Macaulay, J. Algebra 58 (1979), 247-250.

[VV] P. Valabrega and G. Valla, Form rings and regular sequences, Nagoya Math. J. 72 (1978), 93-101.

[Vas] W. V. Vasconcelos, Hilbert functions, analytic spread and Koszul homology, Contemp. Math., vol. 159, Amer. Math. Soc., Providence, RI, 1994, pp. 401-422.

[W] Y. Wu, Reduction number and Hilbert polynomials of ideals in higher dimensional CM local rings, Math. Proc. Cambridge Philos. Soc. 111 (1992), 47.

Department of Mathematics, Purdue University, West Lafayette, Indiana 47907-1395 Current address: Terza Università di Roma, Dipartimento di Matematica, Via Corrado Segre, 2, 00146 Roma, Italy

E-mail address: guerran@venere.mat.uniroma1.it 